\title{
Efficacy of rituximab treatment for thyroid-associated ophthalmopathy as a result of intraorbital B-cell depletion in one patient unresponsive to steroid immunosuppression
}

\author{
Mario Salvi ${ }^{1}$, Guia Vannucchi ${ }^{1}$, Irene Campi ${ }^{1}$, Stefania Rossi ${ }^{5}$, Paola Bonara ${ }^{4}$, Francesco Sbrozzi ${ }^{2}$, \\ Claudio Guastella, Sabrina Avignone ${ }^{3}$, Giacinta Pirola ${ }^{2}$, Roberto Ratiglia ${ }^{2}$ and Paolo Beck-Peccoz ${ }^{1}$ \\ Institutes of ${ }^{1}$ Endocrine Sciences, ${ }^{2}$ Ophthalmology, ${ }^{3}$ Radiology, ${ }^{4}$ Internal Medicine, University of Milan, Fondazione Ospedale Policlinico IRCCS, \\ Via Sforza, 3520122 - Milan, Italy and ${ }^{5}$ Pathology Unit, Department of Medicine, Surgery and Dentistry, Ospedale San Paolo, Milan, Italy \\ (Correspondence should be addressed to P Beck-Peccoz; Email: paolo.beckpeccoz@unimi.it)
}

\begin{abstract}
One patient with Graves' hyperthyroidism and ophthalmopathy in its active phase and unresponsive to steroid, was treated with the anti-CD20 monoclonal antibody, rituximab (RTX), as part of an open study. The effect of RTX in the thyroid and the orbital tissues was studied. The ophthalmopathy responded to RTX therapy by ameliorating the eye signs with a decrease in the clinical activity score from 5 to 2 in 3 months, while the patient had peripheral B-cell depletion. Hyperthyroidism did not improve during the 6 months of B-cell depletion and serum TSH-receptor antibodies (TRAb) levels did not significantly change after RTX therapy. Therefore, the patient underwent total thyroidectomy and few B-cells were found in the thyroid tissue specimens. While the patient eye disease remained stable (clinical activity score $=2$ ), we performed corrective orbital decompression and we found absence of lymphocytes in the orbital tissue specimens. We believe that RTX treatment in Graves' disease may cause amelioration of ophthalmopathy by depleting total lymphocytes population in the orbit. The persistence of Graves' hyperthyroidism suggests that a single cycle of RTX does not result in complete lymphocyte depletion in thyroid tissue and thus no decline in serum TRAb was observed.
\end{abstract}

European Journal of Endocrinology 154 511-517

\section{Introduction}

Graves' disease (GD) is typically a B-cell-mediated autoimmune disease in which hyperthyroidism is caused by stimulating autoantibodies directed against TSH receptor (1) detected in $80-90 \%$ of patients as TSH-binding inhibitory immunoglobulins (TBII) by a recently developed assay $(2,3)$. Serum TSH receptor antibodies (TRAb) usually correlate with the clinical features of GD, being elevated at the onset of hyperthyroidism and also prior to relapse after therapy-induced remission $(4,5)$. It is now accepted that thyroid-associated ophthalmopathy (TAO) results from immunological cross-reactivity between thyroid and orbital tissue antigens, among which the TSH receptor may be involved (6). TSH receptor hyperstimulation in the orbit leads to glycosaminoglycan secretion by pre-adipocytic fibroblasts and subsequent increase of the volume of intraorbital tissues $(7,8)$. It has also been observed that eye muscles $(9,10)$ and connective tissue and fat (11) are characteristically infiltrated by lymphocytes and are target of acute inflammatory reactions which eventually progress to scarring and stabilization of the eye disease (12). Recently, elevated TRAb have been correlated to the clinically active phase of TAO at onset (13) and to response to immunosuppressive therapy (14), although this does not prove a pathogenic role. Rituximab (RTX) is a humanized chimeric anti-CD20 monoclonal antibody whose variable (antigen-binding) region is derived from a mouse antibody. The binding of RTX to the CD20 antigen blocks the activation and differentiation of B-cells, since $\mathrm{CD} 20$ protein is expressed on the surface of pre-B and mature B lymphocytes, but not on stem cells, pro-B lymphocytes and plasma cells (15). Therefore, treatment with RTX leads to specific elimination of B-cells without affecting the regeneration of B-cells from stem cells and the production of immunoglobulins by plasma cells. Graves' hyperthyroidism is caused by B-cell-induced production of TSH receptor-directed immunoglobulins. This fact represents the rationale for patients' therapy with RTX (16). In addition, RTX might be an interesting 
therapeutic option for TAO since it induces transient (46 months) immune suppression with potential efficacy on the characteristic self-limiting, dyphasic inflammatory course of the disease. Here we report a patient, among those included in a phase 3 clinical study on RTX treatment in GD and ophthalmopathy, who was eventually referred for thyroidectomy and orbital decompression to correct ophthalmopathy, allowing immunohistochemistry and cytofluorimetry of both thyroid and orbital tissues after peripheral B-cell depletion. This patient showed a clear improvement of TAO but a marked relapse of hyperthyroidism, during B-cell depletion.

\section{Case report}

A 45-year old woman was diagnosed as having GD in December 2002 and was started on methimazole (MMI) $40 \mathrm{mg} /$ daily and propranolol $20 \mathrm{mg}$ twice daily. Lubricants were prescribed for initial eye inflammatory signs (lid edema and epiphora). The patient also had had type 1 diabetes for 19 years, on insulin therapy (26 Units daily) without $\beta$-cell antigen autoantibodies (anti-GAD, anti-IA2, anti-islet cell, or anti-insulin). On June 2003, the eye examination revealed mild inflammatory signs, Hertel measurement of $21 / 22 \mathrm{~mm}$, no motility dysfunction or neuropathy (NOSPECS 2a 3b 4 $0)(17)$. The diagnosis was compatible with mild active ophthalmopathy, and the patient was euthyroid on $20 \mathrm{mg} /$ daily MMI. She had always been a heavy smoker (20-30 cigarettes a day) and despite being advised so, did not quit after being diagnosed with TAO.

Three months later, on September 2003, she was reevaluated by the ophthalmologist for restriction of the inferior muscles on upgaze in OD. A CT scan showed thickening of all muscles with initial apical crowding in OS and increased inferior and medial recti thickness in OD, but normal optic nerves. The clinical activity score (CAS) was 4 (18) and NOSPECS 2 b 3a 4 a. Shortly after, she was started on methylprednisolone pulse therapy, 16 infusions of $7.5 \mathrm{mg} / \mathrm{kg}$ bw, once a week (November 2003). One month after steroid withdrawal (March 2004) her eye signs worsened with persistent diplopia, Hertel of 24/23 and a more marked restriction of inferior and medial recti muscles bilaterally (CAS of 5, NOSPECS $2 \mathrm{~b} 3 \mathrm{a} 4 \mathrm{~b})$. On ultrasound, she had a thyroid gland slightly larger than normal ( $17.6 \mathrm{ml}$ of volume), with a hypoechoic and disomogeneous structure and no nodules. Since she had not responded to immunosuppression with steroids, we proposed to her the therapy with RTX, which she accepted and was administered on April 14th and 28th (Table 1). On the first infusion the patient reported as a side effect moderate and transient nasal occlusion. At the time of the second infusion, the patient was already reporting improvement of the eye symptoms and diplopia. At ophthalmological examination, decreased inflammation, improved motility, a

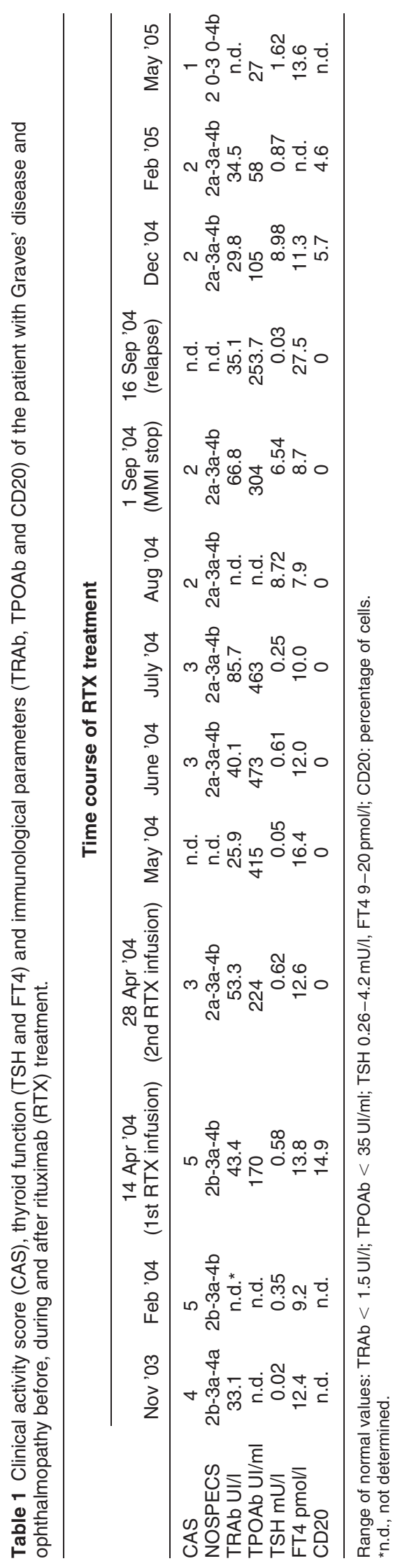


NOSPECS 2a 3a 4 b and a CAS of 3 were found. During the follow-up period after RTX therapy the patient was euthyroid on MMI $10 \mathrm{mg} /$ daily. The patient was seen monthly from June to September 2004, when her CAS decreased to 2 with an unchanged NOSPECS score, eye muscle motility, i.e. restricted inferior and medial recti function, but only inconstant diplopia and normal visual acuity. As assessed by ultrasound, thyroid volume and structure did not change during follow-up. On September 2nd, her serum TSH concentrations were in the hypothyroid range, while on $10 \mathrm{mg}$ MMI, and we withdrew the medication (Table 1). After 2 weeks she was again thyrotoxic, despite the short time off MMI. Radioiodine uptake test was elevated and confirmed the relapse of hyperthyroidism. The patient was also reporting worsening of the diplopia, confirmed at ophthalmological examination, but her CAS remained stable at 2 . The patient was then started again on MMI $5 \mathrm{mg} /$ daily and referred for thyroidectomy, which was performed on November 16th. After surgery the patient was maintained euthyroid on $1.5 \mu \mathrm{g} / \mathrm{kg}$ bw/day of L-thyroxine, while eye disease remained stable $($ CAS $=2)$ afterwards. On February 2005, the patient underwent orbital decompression to correct proptosis. Post-surgical ophthalmological assessment (April 2005) has shown burnt-out disease, with a CAS $=1$. She is now being programmed for surgical correction of diplopia.

\section{Methods}

The patient was seen in our joint thyroid-eye clinic and at each visit blood was taken for thyroid function tests, serum autoantibody testing and for the study of peripheral lymphocytes. Based on clinical findings, thyroid ultrasound was also carried out to assess the goiter changes. The ophthalmological examination included lid fissure and Hertel measurements, color vision, cover test, Hess-Lancaster screen, visual acuity, tonometry, fundus examination and visual field. The severity was classified according to the NOSPECS score (17). Orbital CT scan was performed when the patient begun referring diplopia and subsequently to monitor the effect of therapy and in the preparation of decompressive surgery. At the second ophthalmological visit and at each subsequent examination the CAS was calculated (18), in order to monitor the clinical improvement of the patient. She was then submitted to treatment with RTX, a chimeric human-mouse monoclonal antibody (MabThera) kindly provided by Hoffman La Roche, Basel, Switzerland. The therapeutic protocol recommended was the one used for treatment of patients with rheumatoid arthritis and consisted of i.v. infusion of $1 \mathrm{~g}$ of RTX over approximately $4 \mathrm{hr}$ and $15 \mathrm{~min}$, twice at a 2 -week interval. The study protocol was approved by the ethics committee of the hospital and the patient gave informed consent for the therapy.

\section{Biochemical and immunological testing}

Serum TSH and FT4 concentrations and anti-thyroid antibodies ( $\mathrm{TgAb}$ and TPOAb) were measured using the AutoDELFIA technique (Perkin Elmer-Life Sciences, Wallac Oy, Turku, Finland). Serum TSH receptor autoantibodies, detected as TSH binding inhibitory immunoglobulins (TBII), were measured using a 2nd generation TRAK human lumitest (B.R.A.H.M.S., AG, Henningsdorf/ Berlin, Germany).

\section{Cytofluorimetric analysis}

We looked at the pattern of peripheral blood lymphocytes before therapy and monthly throughout the study period. We also analyzed the lymphocytes within the thyroid tissue, at thyroidectomy, in a lymph node of the neck, removed during surgery, and within the orbital fat and connective tissue, at decompression. We studied the standard immunophenotypic panel (CD3+, $\mathrm{CD} 3+4+, \quad \mathrm{CD} 3+8+, \quad \mathrm{CD} 3+\mathrm{DR}+, \quad \mathrm{CD} 20+$, CD19+5+, CD56+16+3-). Thyroid, lymph node and retrobulbar fat surgical samples were cut in tiny pieces, mashed to become mushy and finally passed many times in a syringe with PBS, until cells were virtually separated and stromas and larger fragments were removed. Aliquots of unstained cell suspension were preliminarily processed in the flowcytometer to evaluate the consistency of cytograms of forward and right scatter. Then, aliquots of around $10^{5}$ lymphocytes were submitted to standard triple staining procedures, in order to carry out immunogating with CD45 and to analyze pairs of monoclonal antibodies to subpopulations of T, B and NK cells, and processed in the flowcytometer (BD Facsan, Cell-quest software, Becton-Dickinson, San Josè, CA, USA).

\section{Histopathology and immunohistochemistry}

Pathological specimens were received fresh and consisted of the total thyroidectomy sample with an excised perithyroid lymph node, and a bilateral incisional biopsy of retrobulbar fat tissue. The thyroidectomy specimen measured $5.3 \times 5.2 \mathrm{~cm}$ in the right lobe, $1.8 \times 1.5 \mathrm{~cm}$ in the isthmus, $4.2 \times 3.2 \mathrm{~cm}$ in the left lobe. The thyroid specimens were first serially and entirely sectioned on a horizontal plane every 3-4 $\mathrm{mm}$ and each slice was examined on its cut surface. Following overnight fixation in buffered 10\% formalin, eight slices for each lobe and two slices including the isthmic area were embedded in paraffin wax, as routine. Each tissue block was examined at different levels by preparing $4 \mathrm{~mm}$-thick paraffin histological sections which were stained with hematoxylin and eosin, as routine for preliminary microscopic evaluation. Additional sections were immunostained using the Biotin Streptavidin Amplified (B-SA) detection system supplied by Bio-Genex, San Ramon, CA, USA, 
and incubated with the following primary antibodies: CD3 (clone F7.2.38; dilution 1:30), CD20 (clone L26; dilution 1:300), CD79a (clone HM57, dilution 1:40, a pan-B marker), CD8 (clone C8/144B; dilution 1:50) all supplied by DAKO Cytomation, Carpinteria, CA, USA, and CD4 (clone 1F6; dilution 1:200) supplied by Novocastra Laboratories Ltd, Newcastle upon Tyne, UK. The excised perithyroid lymph node measured $1.0 \times 0.5 \times 0.4 \mathrm{~cm}$. The sample was bisected and fixed in $10 \%$ formalin overnight then embedded in paraffin wax, as routine. Four mm-thick histological sections were cut from paraffin blocks and stained with hematoxylin and eosin, as routine for preliminary microscopic evaluation. Additional sections were immunostained according to the same procedure and incubated with all the above primary antibodies. Specimens from incisional biopsy of retrobulbar fat tissue measured about $1.0 \times 0.5 \times 0.5 \mathrm{~cm}$ each. They were fixed in formalin and embedded in paraffin, as indicated above. Following the examination of hematoxylin and eosin stained sections, additional sections were immunostained according to the same procedure and incubated with all the above primary antibodies.

\section{Results}

\section{Serum biochemical and immunological findings}

Data are summarized in Table 1. The patient was euthyroid at the beginning of RTX therapy, while kept on $10 \mathrm{mg}$ MMI. Serum TSH concentrations became suppressed two weeks after the second RTX infusion and remained low for about one month, like we have often observed with this treatment (M Salvi, G Vannucchi, I Campi, D Dazzi et al., unpublished observations). Serum TSH concentrations then returned in the normal range and became slightly elevated $(8.7 \mathrm{mU} / \mathrm{l})$ four months after RTX infusion(September 2004), when we decided to withdraw MMI. Serum FT4 concentrations were consistently within the normal range until MMI withdrawal. At the time of relapse of hyperthyroidism, after stopping MMI, we observed both low serum TSH and elevated FT4 concentrations, promptly corrected by restoring MMI therapy. Serum TgAb, TPOAb and TRAb were measured at all times before, during and after RTX therapy. While serum TgAb levels did not change in relation to variations of either thyroid function or therapy, serum TPOAb did show some changes, basically a progressive increase after RTX treatment by doubling their titers for about four months, without any apparent relationship with the clinical situation. TPOAb titers were then declined and became negative within three to four months after thyroidectomy (19). Changes in circulating TRAb bore no relationship to RTX therapy (Table 1). An approximate $50 \%$ reduction of serum TRAb levels was observed at
1-2 weeks after the 2nd infusion of RTX and lasted for 2 months. Then, serum TRAb levels rose to reach a peak of $85 \mathrm{U} / \mathrm{l}$ 4-6 weeks before the relapse of hyperthyroidism. After thyroidectomy, we observed no change of serum TRAb levels which have remained elevated for the following five months. Serum TRAb changes did not correlate with peripheral B-cell depletion, nor with the course of TAO, even after thyroidectomy.

\section{Analysis of lymphocytes subpopulations in peripheral blood and in tissue targets}

Before RTX, the patient's peripheral lymphocyte populations were normal. There were slightly low CD8 + T lymphocytes with normal CD4 + and normal activated T-cells. B lymphocytes, both CD20 + and CD19+, were normal $(14-15 \%)$ with normal co-expression of CD5 + (autoreactive) B-cells (3\%). After the first RTX infusion and for about 6 months (up to October 2004), B-cells were not present in peripheral blood. The absolute numbers of T-cells were also slightly low and the relative percentage distribution of $\mathrm{CD} 4+\mathrm{T}$ cells was increased compared with $\mathrm{CD} 8+$. Data obtained at the time of thyroid surgery are shown in Fig. 1. Peripheral B-cells were repopulating but still low, with $\mathrm{CD} 20+$ lymphocytes about $5.6 \%$ and CD19 3\%, of which about $50 \% \operatorname{CD} 19+5+$. In the thyroid, CD20 were the same as in the blood, whereas CD19 were about twice as many $(7.7 \%)$ of which $50 \%$ were $\mathrm{CD} 19+5+$. In the lymph node there were almost exclusively T-cells. B-cells were as many as in the thyroid and the blood, but CD19 were lower and did not express CD $5+$.

From 7-12 months after RTX infusion peripheral B-cells were still low, at about $5 \%$, with equal percentages of CD20 and CD19 and T-cells remained low in absolute numbers. We were not able to recover either $\mathrm{B}$ or $\mathrm{T}$ lymphocytes from the orbital specimens at the time of decompressive surgery, in keeping with what observed by histology (see below).

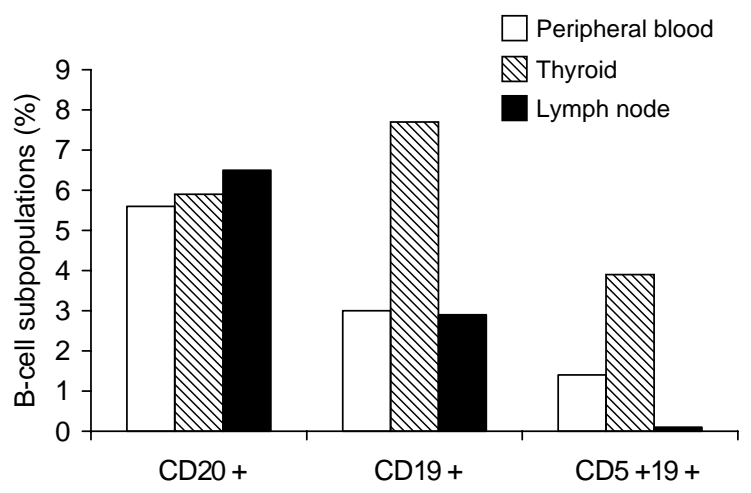

Figure 1 Percentage of B-cell subpopulations in peripheral blood, thyroid and lymph node specimens at the time of thyroidectomy. 


\section{Gross pathology and microscopic findings}

On gross examination, the thyroid parenchyma appeared diffusely homogenous and tan in colour with no nodular lesion. Histologically, the thyroid tissue appeared diffusely hyperplastic, consistent with a diagnosis of diffuse toxic goiter. Multiple tiny lymphoid infiltrates composed of a polymorphous population of small and intermediate sized lymphocytes were observed within the interstitium. Immunostaining showed a prevalence of $\mathrm{CD} 3$ + elements within lymphoid aggregates (Fig. 2A) over the population of $\mathrm{CD} 20+$ and CD79a + (a pan-B marker) B-cells (Fig.2B and C). Histological examination of the lymph node demonstrated a well preserved histoarchitecture with a slight depletion and decrease in the size of germinal centers combined to an expansion of the paracortical compartment. This latter was characterized by the prevalence of small lymphocytes positively immunostained for CD3 (Fig. 2D), with prevalence of $\mathrm{CD} 4+$ over $\mathrm{CD} 8+$ elements in this population (Fig. 2E). Finally, the specimens from bilateral incisional biopsy of retrobulbar fat tissue were characterized histologically by a moderate interstitial edema but no either B- or T-cell infiltration or inflammatory exudate were recorded (Fig. 2F)

\section{Discussion}

This paper reports on the efficacy of immunosuppressive therapy with the anti-CD20 monoclonal antibody RTX in a hyperthyroid patient with TAO, similarly to what recently reported by El Fassi et al. (20). The rationale for this treatment was that TAO might benefit from transient (4-6 months) B-cell depletion induced by RTX, since its natural course has an acute inflammatory phase of 6-12 months duration followed by stabilization of the clinical signs. In addition, since the main pathogenic effector in GD is the TRAb, we thought that B-cell depletion might also affect circulating TRAb and consequently clinical hyperthyroidism. Our main findings in this patient are that during B-cell depletion, while TAO improved progressively and consistently, serum TRAb levels were not affected by RTX treatment and did not decline in relation to the clinical improvement of TAO. By histological and immunohistochemical analysis we were able to show, for the first time, absence of immune cells in the orbit, but not in the thyroid, suggesting that RTX may cause orbital lymphocyte depletion. Hyperthyroidism was well controlled by MMI tapered progressively down to withdrawal in 4 months, when the disease appeared to have reached remission. We could not explain why there was a rise in the titers of TRAb during peripheral B-cell depletion. The lack of effect of RTX on TRAb may have been due to the persistence of plasma cells, which do not express CD20 and may continue antibody production (15). Circulating TRAb could therefore be measured for as long as 4-6 weeks, if one considers that the half life of human $\operatorname{IgG}$ is of approximately 3 weeks (21). In systemic lupus erythematosus, significant peripheral B-cell depletion after RTX has been reported to correlate with clinical disease improvement, but significant changes in anti-dsDNA titers and complement levels
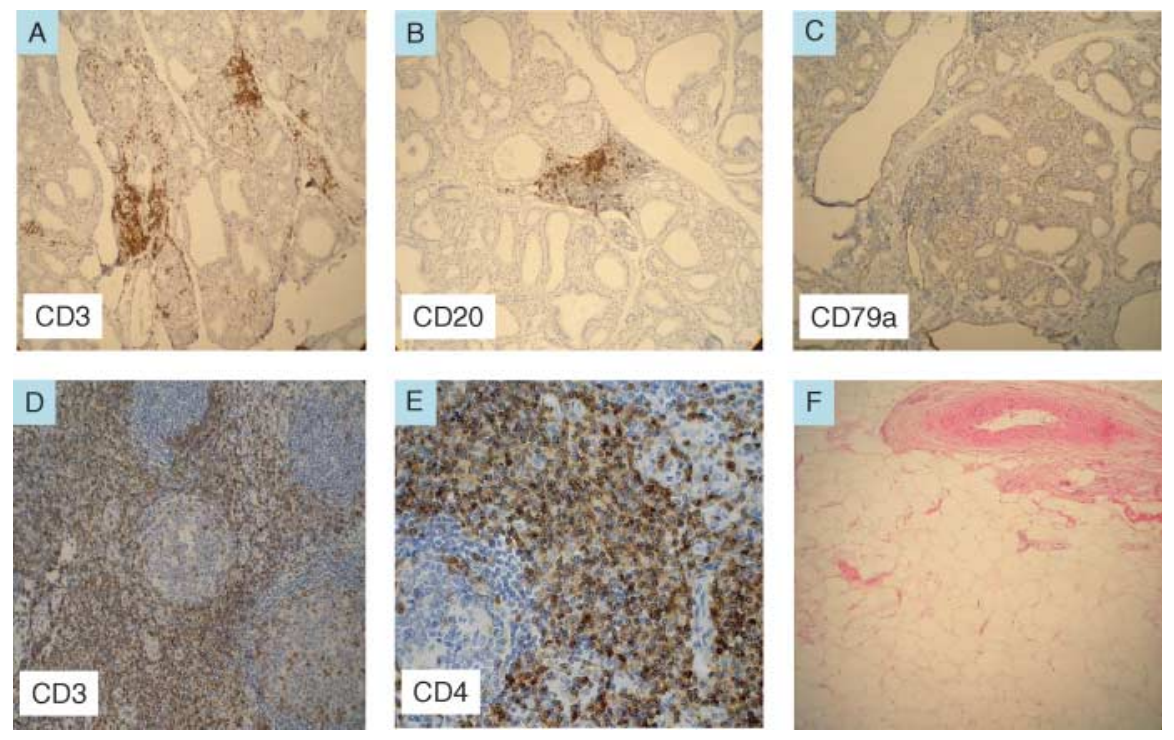

Figure 2 Immunoistochemistry of the thyroid $(A-C)$ and of a lymph node $(D, E)$ at the time of thyroidectomy. Picture A shows small lymphoid infiltrates immunostained for CD3 which are prevalent over CD20 (picture B) and CD79a (picture C) lymphocytes. Picture D shows characteristic germinal centers reduced in size and immunostained for CD3, with prevalence of CD4 lymphocytes (picture E). Picture F shows a low magnification view of orbital fat tissue with the absence of any significant lymphoid infiltrate. Pictures A-C: X200,

counterstained with Hematoxylin; Pictures D and E: X350, counterstained with Hematoxylin; Picture F: X100, Hematoxylin and Eosin. 
were not observed (22). On the other hand, association between the clinical relapse and a rise in autoantibodies, rather than only return of B-cell, has been observed in rheumatoid arthritis (23). Similar results were observed in patients with chronic idiopathic thrombocytopenia, in whom clinical responses after RTX were not associated to significant falls in levels of anti-platelet antibodies (24). The exact mechanism of action for RTX induced B-cell depletion in autoimmune disease is still unclear (15). From the studies conducted in patients with rheumatoid arthritis (25) it seems evident that the clinical state improves progressively over a number of months, despite immediate B-cell depletion after RTX infusion. On the other hand, clinical improvement is sustained even after B-cell return. A key point in our study is that it suggests, for the first time, that besides peripheral B-cell depletion RTX treatment may produce different effects in the target tissues of this organ-specific autoimmune disease, i.e. lymphocyte depletion in the orbit but not in the thyroid. Indeed, in our patient the rise of serum TRAb levels at 5-6 weeks after RTX infusion suggests that autoantibody production may have not occurred in the periphery but in the target organs (26), or perhaps in other lymphoid organs such as the spleen or bone marrow. This is plausible since B lymphocytes were shown to be present in the thyroid specimens at thyroidectomy, both by cytofluorimetry of the tissue homogenates and by immunohistochemistry. The differences in lymphocytic populations of peripheral blood, thyroid and lymph node were consistent with a compartment distribution of the immune response, as expected (27). Although the presence of lymphocytes in thyroid specimens could have been due to initial B-cells repopulation, we cannot exclude that autoreactive B-cells may have still harboured in the thyroid tissue after one course of RTX therapy, and somehow have reactivated after MMI withdrawal. The clinical improvement of TAO after RTX was clearly evident. A delayed response to previous steroid therapy seemed unlikely because the patient had had active and moderate to severe disease for a year and did not seem to respond to such treatment, which had been stopped for about 10 weeks. Her CAS was 5 when RTX therapy was started and decreased to 2-3, consistent with inactive disease, within two weeks after the second RTX infusion. The clinical improvement shortly followed peripheral B-cell depletion two weeks after the first RTX infusion. At the time of decompressive surgery we were surprised to observe the absence of both B and T-cells in the orbit, in contrast with previous findings (9-11). Decompression was carried out about 9 months after RTX therapy, when the patient's CAS was 2-3 and ophthalmopathy was burning out. The orbital tissue specimens were mainly constituted by fat and connective tissue and recently these components have been shown to be infiltrated by T-cells and to be actively involved in the autoimmune reactions (11). Lymphoid infiltration is usually observed in the orbit in disease of long duration (12) and since we cannot explain why both $\mathrm{B}$ and T-cells were not found in the orbit at both cytofluorimetry and immunohistochemistry, we can only interpret, but not prove, that orbital B-cell depletion was the consequence of RTX treatment. Perhaps B-cell depletion has switched off the immune reactivity within the orbit, by inhibiting B lymphocyte antigen presentation (15) and T cell activation, as was shown in rheumatoid arthritis $(28,29)$. Although we do not clearly know the pathogenic reactions underlying the orbital involvement in TAO, evidence has been found to support a role of TRAb (6). The results of the present work are not consistent with this hypothesis, since the clinical improvement of TAO and the lymphocyte depletion in the orbit did not correlate with any changes in serum TRAb. Recently, Smith and colleagues (8) have found that antibodies to the IGF-I receptor expressed on fibroblasts induce production of IL-16 and RANTES, that act as chemoattractants for T-cells within the orbital connective tissue and have proposed a potential common pathogenic pathway in rheumatoid arthritis synovial fibroblasts (30).

Interestingly, although RA has classically been thought of as a predominantly T-cell mediated disease (23), RTX is effective in producing clinical improvement by depleting B-cells but not a consistent decline in serum rheumatoid factor levels (28). Because of the self-limiting clinical course of TAO, RTX represents an ideal therapy also in consideration of the paucity of known side effects. Indeed, TAO in our patient did improve with RTX treatment and remained stable thereafter even after peripheral B-cell return, allowing orbital decompression and proper ophthalmomanagement.

\section{Acknowledgements}

We wish to thank Dr Martino Introna of the Division of Haematology, Ospedali Riuniti, Bergamo, Italy for fruitful discussion on the rationale of the work and Dr Tim Shaw of Hoffman La Roche, England for providing us with Mab Thera. This work is supported in part by MURST, Roma and by Fondazione Ospedale Policlinico, IRCCS, Milano, Italy.

\section{References}

1 Zakarija M \& McKenzie JM. The spectrum and significance of autoantibodies reacting with the thyrotropin receptor. Endocrinology and Metabolism Clinics of North America 198716 343-363.

2 Costagliola S, Morgenthaler NG, Hoermann R, Badenhoop K, Struck J, Freitag D, Poertl S, Weglohner W, Hollidt JM, Quadbeck B, Dumont JE, Schumm-Draeger PM, Bergmann A, Mann K, Vassart G \& Usadel KH. Second generation assay for thyrotropin receptor antibodies has superior diagnostic sensitivity for Graves' disease. Journal of Clinical Endocrinology and Metabolism $19998490-97$. 
3 Maugendre D \& Massart C. Clinical value of a new TSH binding inihibitory activity assay using human TSH receptors in the follow-up of antithyroid drug treated Graves' disease. Comparison with thyroid stimulating antibody bioassay. Clinical Endocrinology $20015489-96$.

4 Rees Smith B, McLachlan SM \& Furmaniak J. Autoantibodies to the thyrotropin receptor. Endocrine Review $1988 \mathbf{8} 106-121$.

5 Michelangeli V, Poon C, Taft J, Newnham H, Topliss D \& Colman P. The prognostic value of thyrotropin receptor antibody measurement in the early stages of treatment of Graves' disease with antithyroid drugs. Thyroid $1998 \mathbf{8} 119-124$.

6 Bahn RS. Clinical review 157: Pathophysiology of Graves' ophthalmopathy: the cycle of disease. Journal of Clinical Endocrinology and Metabolism 200388 1939-1946.

7 Valyasevi RW, Harteneck DA, Dutton CM \& Bahn RS. Stimulation of adipogenesis, peroxisome proliferator-activated receptorgamma (PPARgamma), and thyrotropin receptor by PPARgamma agonist in human orbital preadipocyte fibroblasts. Journal of Clinical Endocrinology and Metabolism 200287 2352-2358.

8 Cao HJ, Wang HS, Zhang Y, Lin HY, Phipps RP \& Smith TJ. Activation of human orbital fibroblasts through CD40 engagement results in a dramatic induction of hyaluronan synthesis and prostaglandin endoperoxide $\mathrm{H}$ synthase-2 expression: insights into potential pathogenic mechanisms of thyroid-associated ophthalmopathy. Journal of Biological Chemistry 1998273 29615-29625.

9 Hufnagel TJ, Hickey WF, Cobbs WH, Jakobiec FA, Iwamoto T \& Eagle RC. Immunohistochemical and ultrastructural studies on the exenterated orbital tissues of a patient with Graves' disease. Ophthalmology $1984911411-1419$.

10 Weetman AP, Cohen S, Gatter KC, Fells P \& Shine B. Immunohistochemical analysis of the retrobulbar tissues in Graves' ophthalmopathy. Clinical and Experimental Immunology $1989 \quad \mathbf{7 5}$ $222-227$

11 Eckstein AK, Quadbeck B, Tews S, Mann K, Kruger C, Mohr CH, Steuhl KP, Esser J \& Gieseler RK. Thyroid-associated ophthalmopathy: evidence for CD4 $(+)$ gammadelta T-cells; de novo differentiation of RFD7 $(+)$ macrophages, but not RFD1 $(+)$ dendritic cells, and loss of gammadelta and alphabeta $\mathrm{T}$ cell receptor expression. British Journal of Ophthalmology 200488 803-808.

12 Pappa A, Lawson JM, Calder V, Fells P \& Lightman S. T-cells and fibroblasts in affected extraocular muscle in early and late thyroid-associated ophthalmopathy. British Journal of Ophthalmology $2000 \mathbf{8 4} 517-522$.

13 Gerding MN, van der Meer JW, Broenink M, Bakker O, Wiersinga WM \& Prummel MF. Association of thyrotrophin receptor antibodies with the clinical features of Graves' ophthalmopathy. Clinical Endocrinology 200052 267-271.

14 Eckstein AK, Plicht M, Lax H, Hirche H, Quadbeck B, Mann K, Steuhl KP, Esser J \& Morgenthaler NG. Clinical results of antiinflammatory therapy in Graves' ophthalmopathy and association with thyroidal autoantibodies. Clinical Endocrinology $2004 \mathbf{6 1}$ 612-618.

15 Tsokos GC B-cells. Be Gone, B-Cell Depletion in the Treatment of Rheumatoid Arthritis. New England Journal of Medicine 2004 $3502546-2548$.

16 Hasselbalch HC. B-cell depletion with rituximab - a targeted therapy for Graves' disease and autoimmune thyroiditis. Immunology Letters $2003 \mathbf{8 8} 85-86$.
17 Werner SC. Classification of the eye changes of Graves' disease. Journal of Clinical Endocrinology and Metabolism 196929 982-984.

18 Mourits MP, Prummel MF, Wiersinga WM \& Koorneef L. Clinical activity score as a guide in the management of patients with Graves' ophthalmopathy. Clinical Endocrinology 1997 47 9-14.

19 Chiovato L, Latrofa F, Braverman LE, Pacini F, Capezzone M, Masserini L, Grasso L \& Pinchera A. Disappearance of humoral thyroid autoimmunity after complete removal of thyroid antigens. Annals of Internal Medicine $2003139346-351$.

20 El Fassi D, Nielsen CH, Hasselbalch HC \& Hegedus L. Successful B lymphocyte depletion in a patient with recurrent Graves' disease and severe ophthalmopathy. Thyroid 200515 (Suppl 1) S28 (Abstract).

21 Male D, Cooke A, Owen M, Trowsdale J \& Champion B. Antigen receptor molecules. In Advanced Immunology, Eds. D Male, A Cooke, M Owen, J Trowsdale \& B Champion. pp 2.1-2.2. London: Mosby, 1996.

22 Anolik JH, Campbell D, Felgar RE, Rosenblatt J, Young F, Sanz I \& Looney RJ. B lymphocyte depletion in the treatment of systemic lupus erythematosus. Arthritis and Rheumatism 2002 (Suppl 9) S717 (Abstract).

23 Edwards JC, Szczepanski L, Szechinski J, Filipowicz-Sosnowska A, Emery P, Close DR, Stevens RM \& Shaw T. Efficacy of B-celltargeted therapy with rituximab in patients with rheumatoid arthritis. New England Journal of Medicine $20043 \mathbf{3 0 0} 2572-2581$.

24 Stasi R, Pagano A, Stipa E \& Amadori S. Rituximab chimeric antiCD20 monoclonal antibody treatment for adults with chronic idiopathic thrombocytopenic purpura. Blood $200198952-957$.

25 Leandro MJ, Edwards JC \& Cambridge G. Clinical outcome in 22 patients with rheumatoid arthritis treated for B lymphocyte depletion. Annals of the Rheumatic Diseases 200261 883-888.

26 Segundo C, Rodriguez C, Garcia-Poley A, Aguilar M, Gavilan I, Bellas C \& Brieva JA. Thyroid-infiltrating B lymphocytes in Graves' disease are related to marginal zone and memory B-cell compartments. Thyroid $200111525-530$.

27 Armengol MP, Juan M, Lucas-Martin A, Fernandez-Figueras MT, Jaraquemada D, Gallart T \& Pujol-Borrell R. Thyroid autoimmune disease: demonstration of thyroid antigen-specific B-cells and recombination-activating gene expression in chemokine-containing active intrathyroidal germinal centers. American Journal of Pathology $2001159861-873$.

28 Gorman C, Leandro M \& Isenberg D. B-cell depletion in autoimmune disease. Arthritis Research and Therapy 20035 (Suppl 4) S17-S21.

29 Takemura S, Klimiuk PA, Braun A, Goronzy JJ \& Weyand CM. $\mathrm{T}$ cell activation in rheumatoid synovium is B-cell dependent. Journal of Immunology $2001 \mathbf{1 6 7}$ 4710-4718.

30 Pritchard J, Tsui S, Horst N, Cruikshank WW \& Smith TJ. Synovial fibroblasts from patients with rheumatoid arthritis, like fibroblasts from Graves'disease, express high levels of IL-16 when treated with Igs against insulin-like growth factor-1 receptor. Journal of Immunology $20041733564-3569$.

Received 7 October 2005

Accepted 12 January 2006 